\title{
Educación Médica de Postgrado Estándares globales de la WFME para la mejora de la calidad
}

\section{EL PROYECTO DE LA WFME SOBRE ESTÁNDARES EN EDUCACIÓN MÉDICA DE POSTGRADO}

Para desarrollar el proyecto sobre Estándares Internacionales en Educación Médica en la educación médica de postgrado, la WFME, en junio de 2001, designó un Comité Internacional consistente en un Grupo de Trabajo y un Consejo Internacional de Expertos, encargados de definir estándares internacionales para programas educativos en educación médica de postgrado.

Las deliberaciones del Grupo de Trabajo, que se reunieron en Copenhague en septiembre de 2001, se basaron en materiales de diferentes orígenes ${ }^{8-12}$. En su informe, el Grupo de Trabajo definió una serie de estándares internacionales en educación médica de postgrado, diseñados para animar a las instituciones de educación médica de postgrado en diferentes niveles de desarrollo, y con diferentes condiciones educativas, socioeconómicas y culturales diferentes, a utilizar el sistema de estándares a un nivel apropiado para ellas. El énfasis se puso en el hecho de que los estándares funcionasen como una palanca para el cambio y la reforma.

El proyecto se revisó en base a los comentarios recibidos del Grupo Internacional de Expertos y de las conferencias internacionales donde se presentaron los resultados.

El documento final fue aprobado por el Comité Ejecutivo de la WFME en septiembre de 2002.

\section{FUNDAMENTOS DE LA EDUCACIÓN MÉDICA DE POSTGRADO}

La educación médica de postgrado es la fase de la educación médica en que los médicos desarrollan competencias después de haber acabado la educación médica básica o de pregrado. Esta parte de la formación se desarrolla según normas y regulaciones específicas. La formación se desarrolla a partir de una estructura similar a la del aprendizaje, en la cual los médicos jóvenes en situaciones clínicas, están bajo la supervisión de colegas con más experiencia que asumen la responsabilidad de su formación e instrucción.
La educación médica de postgrado comprende la formación de la especialidad y subespecialidad, la medicina familiar y otros programas reglados de formación en diferentes áreas de experteza.

Además de los aspectos clínicos prácticos, se requiere cierta educación teórica. Esta se puede organizar de diferentes maneras, bien sea ligada a la formación clínica o a través de cursos teóricos regionales, nacionales o internacionales. Estos programas serían organizados por las universidades, comités de especialidad, sociedades médicas y colegios o institutos para la educación médica de postgrado.

La educación médica de postgrado es parte de la formación continuada en medicina, que también incluye la Educación Médica Continuada (EMC) o Desarrollo Profesional Continuado (DPC). La EMC/DPC se caracterizan por el aprendizaje autodirigido, más que por la formación supervisada. Todo y que a menudo se utilizan para designar el período que comienza después de completar la formación de pregrado o postgrado, es evidente que la EMC/DPC es una actividad que va más allá en el "continuum" de la educación médica.

Internacionalmente, hay variaciones considerables en el número de especialidades reconocidas y áreas de experteza en medicina y en la organización, la estructura, el contenido y los requerimientos en la educación médica de postgrado. Las calificaciones en áreas de experteza también se obtienen a través de la EMC. En algunas regiones del mundo, la formación especializada tiene lugar a través de contratos en departamentos hospitalarios/instalaciones sanitarias durante algunos años, mientras que en otros lugares hay cursos teóricos de corta duración sin requerimientos específicos de formación práctica.

De todas maneras, en las últimas décadas ha habido una convergencia creciente en métodos de formación haciendo énfasis en la formación práctica y teórica. Los principios modernos de educación médica han ejercido una influencia creciente en todos los países. En la educación médica de postgrado se han desarrollado programas de formación muy sofisticados, los cuales constan de formación clíni- 
ca/práctica, supervisión de expertos, enseñanza teórica, investigación, evaluación sistemática y de los programas de formación.

La convergencia de los principios de la formación de postgrado en todo el mundo se ha promovido gracias a una mejora de la comunicación entre universidades/instituciones educativas, cuerpos reguladores, sociedades médicas y asociaciones médicas. Estas también han estado influenciadas por la mayor movilidad de los médicos y la internacionalización creciente de la medicina, con el soporte de acuerdos que permiten el intercambio internacional de médicos entre diferentes partes del mundo, p.e. EU, NAFTA y MERCOSUR. La importancia de este desarrollo para la profesión médica está documentada en Europa a través de la adopción de las Directivas para los Médicos ${ }^{13}$ y el trabajo del Comité Asesor de Formación Médica de la Comisión Europea $^{14}$. Por tanto se refuerza la necesidad de un sistema internacional de garantía de calidad en la educación médica de postgrado.

\section{CONCEPTO, OBJETIVOS Y RACIONALIDAD}

Es posible definir estándares internacionales de aplicación general para la educación médica ${ }^{5}$. Estos tienen en cuenta las variaciones interculturales en educación médica, debidas a diferencias en las tradiciones docentes, culturales, condiciones socioeconómicas, el espectro de salud y enfermedad, y formas diferentes de proveer la asistencia sanitaria. Estas diferencias también pueden darse dentro de un mismo país. Las bases científicas de la medicina son universales. La tarea de la educación médica en todos los sitios, es proveer atención sanitaria. A pesar de estas variaciones, hay un alto nivel de equivalencias estructurales, de proceso y de producto de la educación médica de postgrado en todo el mundo.

Los estándares internacionales, por supuesto, han de ser modificados o complementados de acuerdo con las necesidades y prioridades regionales, nacionales e institucionales. Cada país tiene la responsabilidad de garantizar que su programa de formación médica de postgrado dé soporte a objetivos sanitarios nacionales.

La WFME también ha dejado bien claro que no pueden haber beneficios en fomentar la uniformidad de los programas educativos ${ }^{5}$. Y aún más, la garantía de calidad de los programas de formación médica ha de enfatizar la mejora, y ser una guía para alcanzar desarrollos para evitar interpretar los estándares como un sistema de igualar a un nivel más bajo de calidad.

Una parte nuclear de la estrategia de la WFME es dar prioridad a las especificaciones de los estándares internacionales y pautas para la educación médica, incluyendo ambos a las instituciones y sus productos educativos. La adopción de los estándares internacionales constituirá un nuevo marco para las autoridades, organizaciones e instituciones responsables de la educación médica de postgrado, como instrumento de medida de ellas mismas. Aún más, los estándares aceptados internacionalmente podrían ser usados como base para el reconocimiento nacional y regional y la acreditación de programas educativos de postgrado.

La evaluación basada en estándares aceptados generalmente es un incentivo importante para la mejora y para incrementar la calidad de la educación médica, cuando se pretende una reordenación y una reforma y también para promover una mejora continua y un desarrollo.

La adopción de estándares aceptados internacionalmente tiene el potencial de sentar las bases para la evaluación nacional de la educación médica de postgrado así como un reconocimiento regional más amplio.

Los estándares globales compartidos en educación médica facilitarán la movilidad de los médicos, y facilitarán la aceptación de médicos en países diferentes a los que se han formado. Se facilitará por tanto el mantenimiento de las competencias de los médicos que se han formado en otros países.

Finalmente, los programas de formación subestándares se pueden mejorar con el uso de un sistema de evaluación y acreditación basado en estándares aceptados internacionalmente, mejorando así la calidad de los sistemas de salud tanto a nivel nacional como internacional.

\section{PREMISAS PARA LOS ESTÁNDARES DE POSTGRADO}

El Grupo de Trabajo de la WFME aplicó los principios que se desarrollaron sobre la educación médica básica ${ }^{6}$ a la educación médica de postgrado. La atención se centró en la aplicación general de las directivas para el desarrollo de la calidad de la educación médica. Se adoptaron las siguientes premisas para que los estándares internacionales en educación médica de postgrado fueran aceptados universalmente:

- Sólo se habrían de cubrir aspectos generales de la educación médica de postgrado. 
- Los estándares habrían de englobar categorías amplias en contenidos, proceso, ámbito educativo y resultado de la educación médica de postgrado.

- Los estándares habrían de ser útiles para el cambio y la reforma.

- Los estándares no sólo han de servir para establecer requerimientos globales mínimos, sino también para estimular el desarrollo de la calidad, más allá de los niveles especificados.

- Los estándares habrían de estar formulados de forma tal que permitieran las diferencias nacionales y regionales en los programas educativos, y los diferentes perfiles y desarrollos a nivel local, nacional y regional.

- El cumplimiento de los estándares ha de ser responsabilidad de cada comunidad, país o región.

- El uso de un grupo común de estándares internacionales no implica o requiere una equivalencia completa del contenido de los programas y resultados de la educación médica de postgrado, pero las desviaciones tendrían que ser definidas claramente y razonadas.

- Los estándares deberían reconocer la naturaleza dinámica del desarrollo del programa.

- Los estándares están formulados como un instrumento que las autoridades responsables para la educación médica de postgrado, pueden usar para su desarrollo institucional y su programa educativo.

- Los estándares no han de ser usados para establecer un ránquing de instituciones.

- Los estándares se deberían continuar desarrollando con discusiones y consenso internacional.

- El valor de los estándares se ha de demostrar con estudios evaluativos en cada región.

Los estándares han de estar claramente definidos, ser lógicos, apropiados, relevantes, mensurables, realizables y aceptables por los usuarios. Han de tener consecuencias prácticas, reconocer la diversidad y fomentar un desarrollo adecuado.

\section{USO DE LOS ESTÁNDARES}

La WFME opina que los estándares internacionales presentados pueden ser usados globalmente como un instrumento para garantizar la calidad y el desarrollo de la educación médica de postgrado. Esto puede llevarse a cabo de diferentes formas como:

- Autoevaluación institucional. La intención principal de la WFME al introducir un instrumento para la mejora de la calidad, es proveer un nuevo marco en el cual las autoridades con responsabili- dad en la educación médica de postgrado pueden valorarse ellas mismas a través de procesos de autoevaluación institucional voluntaria y establecer procesos de automejora. Las pautas pueden ser consideradas como un Manual de Autoevaluación.

- Revisión externa. Este proceso aún se puede desarrollar más, incluyendo la evaluación y el consejo de comités de revisión externos.

- Combinación de autoevaluación institucional y revisión externa. La WFME considera que esta combinación es el método más válido.

- Reconocimiento y acreditación. Dependiendo de las necesidades y tradiciones locales, las pautas también pueden ser usadas por agencias nacionales o regionales encargadas del reconocimiento y acreditación de la educación médica de postgrado.

\section{DEFINICIONES}

La educación médica de postgrado se podría definir como la fase en la cual los médicos se forman bajo supervisión para una práctica independiente después de completar su formación médica básica. Consta de formación de especialista y subespecialista, medicina familiar y otros programas de formación reglados. Después de completar un programa de formación de postgrado reglado, normalmente se otorga un diploma o certificado.

Todo y que la educación médica de postgrado es una fase limitada en el continuum de la educación médica, no se puede separar claramente de la Educación Médica Continuada (EMC) o del Desarrollo Profesional Continuo (DPC). Estos llevan se llevan a cabo durante toda la vida profesional después de la licenciatura en la facultad y se caracterizan por el aprendizaje autodirigido y pocas veces comportan formación supervisada durante largos períodos de tiempo.

La WFME recomienda el siguiente grupo de estándares globales de la educación médica de postgrado estructurados de acuerdo con 9 áreas y 38 subáreas.

Las ÁREAS definidas como componentes amplios se refieren a la estructura, proceso y resultado de la educación médica de postgrado y formación cubren:

1. Objetivos y resultados

2. Proceso de formación

3. Evaluación de los residentes

4. Residentes

5. Plantilla académica

6. Marco para la formación y recursos educativos

7. Programa evaluativo 
8. Gobernabilidad y gestión

9. Renovación continuada

Las SUBÁREAS se definen como aspectos específicos de una área, correspondientes a indicadores de actuación.

Los ESTÁNDARES se especifican para cada área utilizando dos niveles de adquisición:

- Estándares básicos. Los estándares se han de aplicar y su cumplimiento ha de quedar de manifiesto en la evaluación del programa de formación. Los estándares básicos se enuncian con la expresión "se debe".

- Estándares para el desarrollo de la calidad. Son los que reflejan el consenso internacional sobre la mejor práctica para la educación médica de postgrado. El cumplimiento de estos estándares dependerá del estadio de desarrollo del programa de formación, sus recursos, la política educativa y otras condiciones locales que influyan en la relevancia y las prioridades. Incluso los programas más avanzados no pueden cumplir todos los estándares. Los estándares para el desarrollo de la calidad, se enuncian con la expresión "debería".

Las ANOTACIONES se utilizan para clarificar, ampliar o ejemplificar los términos utilizados.

\section{MISIÓN Y RESULTADOS}

\subsection{FORMULACIÓN DE LA MISIÓN Y DE LOS RESULTADOS}

\section{Estándares básicos}

Las autoridades competentes deben definir, consultadas las organizaciones profesionales, la misión y los resultados que se esperan para los diferentes tipos de formación médica de postgrado, y hacerlos públicos. La formulación de la misión y de los resultados debe describir el proceso educativo del que ha de resultar un médico competente para ejercer una práctica médica amplia y actualizada en el campo de la medicina de una forma profesional, sin supervisión ya sea de forma independiente ya sea en el seno de un equipo, y de acuerdo con las funciones que los médicos pueden ejercer en el sistema sanitario.

\section{Desarrollo de la calidad}

La misión y los objetivos deberían estimular una innovación apropiada en el proceso de formación y permitir el desarrollo de las competencias más amplias de las requeridas mínimamente, y persistir constantemente para mejorar la atención del paciente que sea apropiada, efectiva y compasiva, para afrontar los problemas de salud y la promoción de la salud. La formación debería animar a los médicos a convertirse en expertos en su especialidad y debería prepararlos para un aprendizaje autodirigido a lo largo de la vida y estar a punto para proseguir la educación médica y el desarrollo profesionales.

\section{Anotaciones:}

- La formulación de la misión y de los resultados incluiría aspectos generales y específicos relevantes para las políticas nacionales y regionales.

- Las autoridades competentes incluirían organizaciones locales y nacionales implicados en la regulación de la educación médica de postgrado y podría ser una agencia gubernamental nacional, un consejo nacional, una universidad, una organización profesional competente o una combinación de varias de ellas.

- Los tipos de formación médica de postgrado incluirían la residencia, la formación sistemática vocacional, la formación especializada y otras formas regladas de formación para alcanzar la experteza en áreas específicas de la medicina.

- Convertirse en expertos se refiere a un compromiso más profundo y/o amplio en el desarrollo de la disciplina, incluyendo la responsabilidad para la educación, desarrollo, investigación y gestión.

- La especialidad incluiría especialidades reconocidas, incluyendo medicina general, subespecialidades y otras áreas de experteza.

\subsection{PARTICIPACIÓN EN LA FORMULACIÓN DE LOS OBJETIVOS Y DE LOS RESULTADOS}

\section{Estándares básicos}

La formulación de la misión y de los resultados de la formación de postgrado debe ser hecha por sus agentes principales.

\section{Desarrollo de la calidad}

La formulación de la misión y de los resultados debería basarse en aportaciones de un colectivo más amplio de agentes sociales.

\section{Anotaciones:}

- Los agentes principales serían los residentes, los directores de programas, las sociedades científicas, las administraciones hospitalarias, las autoridades gubernamentales y las asociaciones ú organizaciones profesionales.

- El colectivo más amplio de agentes sociales incluiría una representación de los supervisores, profesores, tutores, otros profesionales sanitarios, pacientes, la comunidad, organizaciones y autoridades sanitarias. 


\subsection{PROFESIONALIDAD Y AUTONOMÍA}

\section{Estándares básicos}

El proceso de formación, basado en la educación médica básica reconocida, debe reforzar aún más la profesionalidad del médico.

\section{Desarrollo de la calidad}

La formación debería fomentar la autonomía profesional para permitir al médico actuar en el mejor interés del paciente y del público.

\section{Anotaciones:}

La profesionalidad describe el conocimiento, las habilidades, las actitudes y los comportamientos que los pacientes y la sociedad esperan de los individuos durante el ejercicio de su profesión e incluye conceptos como la capacidad de aprender a lo largo de la vida y el mantenimiento de la competencia, mantenerse informado, el comportamiento ético, la integridad, la honestidad, el altruismo, el servicio a los otros, la adhesión a los códigos profesionales, la justicia y el respeto a los otros.

\subsection{RESULTADOS DEL PROCESO FORMATIVO}

\section{Estándares básicos}

Las autoridades competentes relevantes, de acuerdo con las organizaciones profesionales, deben definir las competencias que han de adquirir los residentes como resultado de los programas de formación.

\section{Desarrollo de la calidad}

Se debería especificar tanto las competencias más generales como las más específicas que deben adquirir los residentes, y su relación con las competencias obtenidas como resultado de la educación médica básica. La medida de las competencias obtenidas por los residentes se debería utilizar como feedback para el desarrollo del programa.

\section{Anotaciones:}

- Las competencias se pueden definir en términos profesionales amplios como un conocimiento específico, habilidades, actitudes y comportamientos. Las competencias relevantes para la formación de postgrado incluirían, dependiendo de la especialidad escogida, las siguientes áreas:

- El cuidado del paciente que sea apropiado, efectivo y compasivo para afrontar los problemas de salud y la promoción de la salud.

- El conocimiento médico en las ciencias biomédicas, clínicas y de comportamiento, y la ética y jurisprudencia médicas, y la aplicación este conocimiento al cuidado del paciente.

- Las aptitudes interpersonales y las habilidades de comunicación que garanticen un intercambio efectivo de comunicación con pacientes individuales y sus familias, y un trabajo de equipo con otros profesionales sanitarios, la comunidad científica y el público.

- La valoración y utilización de nuevo conocimiento científico para actualizar continuadamente y mejorar la práctica clínica.

- La función como supervisor, formador y profesor en relación a los colegas, a los estudiantes de medicina y de otros profesionales sanitarios

- La capacidad de ser experto contribuyendo al desarrollo y la investigación del campo médico escogido.

- La profesionalidad.

- El interés y la capacidad de actuar como un defensor del paciente

- El conocimiento de la salud pública y de las políticas de salud y el conocimiento y la respuesta al contexto más amplio del sistema sanitario, incluyendo, p.e. la organización de la atención, la alianza con proveedores y gestores de la atención sanitaria, la práctica de una atención con una buena relación coste-efectividad, la economía de la salud y la ubicación de recursos.

- La capacidad de entender la atención sanitaria, e identificar y llevar a cabo una mejora de la atención basada en el sistema.

\section{PROCESO DE FORMACIÓN}

\subsection{METODOLOGÍAS DE APRENDIZAJE}

\section{Estándares básicos}

La formación médica de postgrado debe seguir un programa formativo sistemático, que describa los componentes genéricos y específicos para cada disciplina. La formación debe ser práctica, haciendo participar al residente en los servicios y tareas de atención al paciente en las instituciones docentes. El programa de formación debe integrar la práctica con la teoría.

\section{Desarrollo de la calidad}

La formación médica de postgrado debería ser una interfase entre la educación médica básica y la educación médica continuada/desarrollo profesional. La formación debería ser dirigida y el residente debería ser guiado, mediante la supervisión y el asesoramiento regular. El proceso de formación debería garantizar una independencia creciente a medida que mejoran las habilidades, el conocimiento y la experiencia. Cada residente debería tener acceso al consejo educativo. 


\section{Anotaciones:}

- El proceso de formación se desarrollaría a través de un tronco común, desde un contenido general a uno más especializado.

- El consejo educativo incluiría el acceso a tutores o mentores designados.

\subsection{MÉTODOS CIENTÍFICOS}

\section{Estándares básicos}

El residente debe adquirir el conocimiento de las bases científicas y los métodos de la especialidad escogida de la medicina, y a través de la exposición a un abanico amplio de experiencia clínica/práctica relevante en diferentes ámbitos del campo escogido de la medicina, debe familiarizarse con la medicina basada en la evidencia y el proceso de decisiones clínicas críticas.

\section{Desarrollo de la calidad}

Durante el proceso de formación el residente debería tener una instrucción formal sobre la documentación médica, el análisis de informes científicos y la medicina basada en la evidencia, y debería involucrarse en investigación.

\section{Anotaciones:}

La formación en las bases y los métodos científicos podría incluir la participación de proyectos de investigación optativos por parte de los residentes (cf.6.5)

\subsection{CONTENIDO DEL PROCESO DE FORMACIÓN}

\section{Estándares básicos}

El proceso de formación debe incluir la práctica clínica y los aspectos relevantes de las ciencias biomédicas básicas, clínicas, sociales y del comportamiento; el proceso de la decisión clínica; las habilidades de comunicación, la ética médica, la política de salud pública, la jurisprudencia médica y la disciplina para la gestión necesarias para demostrar una práctica profesional en el campo escogido de la medicina.

\section{Desarrollo de la calidad}

El proceso de formación debería garantizar el desarrollo del conocimiento, las habilidades, las actitudes y los atributos personales en las funciones de experto médico, promotor de salud, comunicador, colaborador y trabajador en equipo, académico, administrador y gestor.

\section{Anotaciones:}

- Las ciencias biomédicas básicas incluirían -dependiendo de las necesidades locales, intereses y tradiciones, anatomía, bioquímica, fisiología, física médica, biología celular y molecular, genética, microbiología, inmunología, farmacología, patología, etc.

- Las ciencias clínicas incluirían las disciplinas clínicas o de laboratorio escogidas, además de otras disciplinas clínicas/de laboratorio relevantes.

- Las ciencias sociales y de comportamiento incluirían -dependiendo de las necesidades locales, intereses y tradiciones-, psicología y sociología médicas, bioestadística, epidemiología, higiene y salud pública, medicina comunitaria, etc.

- Las ciencias sociales y de comportamiento y la ética médica deberían proporcionar el conocimiento, los conceptos, los métodos, las habilidades y las actitudes necesarios para entender los determinantes socioeconómicos, demográficos y culturales de las causas, distribución y consecuencias de los problemas de salud.

\subsection{ESTRUCTURA, COMPOSICIÓN Y DURACIÓN DE LA FORMACIÓN}

\section{Estándares básicos}

La composición global, la estructura y la duración del proceso de formación y el desarrollo profesional deben estar descritos con una clara definición de los objetivos y los resultados deseados, basados en las tareas, y la explicación de su relación con la educación médica básica y la atención sanitaria. Se deben especificar claramente cuales son los componente opcionales y cuales los obligatorios.

\section{Desarrollo de la calidad}

Se debería garantizar en el proceso de formación la integración de la teoría y de la práctica.

\section{Anotaciones:}

- La estructura de la formación hace referencia a la secuencia global de vínculo a las estructuras de formación y a la responsabilidad del médico, y no a los detalles de las experiencias durante la formación.

- La integración de la práctica con la teoría incluiría sesiones de aprendizaje didácticas y experiencias supervisadas de atención al paciente.

\subsection{LA RELACIÓN ENTRE LA FORMACIÓN Y EL SERVICIO}

\section{Estándares básicos}

Se debe describir y respetar la naturaleza de aprendizaje del desarrollo profesional y se debe garantizar la integración entre formación y servicio (formación en el trabajo). 


\section{Desarrollo de la calidad}

La capacidad del sistema sanitario debería ser utilizada efectivamente con objetivos de formación basados en el servicio. La formación impartida debería ser complementaria y no subordinada a las demandas del servicio.

\section{Anotaciones:}

- La integración entre la formación y el servicio implica de una parte la oferta de un buen servicio sanitario por parte de los residentes, y por otra que las oportunidades para aprender estén incluidas dentro de las funciones de servicio.

- La utilización efectiva se refiere a la optimización del uso de diferentes ámbitos clínicos, al uso de pacientes y situaciones clínicas con objetivos de formación respetando al tiempo las funciones de servicio.

\subsection{GESTIÓN DE LA FORMACIÓN}

\section{Estándares básicos}

Se deben identificar claramente la responsabilidad y la autoridad para organizar, coordinar, dirigir y evaluar la formación individual y el proceso de formación.

\section{Desarrollo de la calidad}

Se debería garantizar una formación coordinada con diferentes áreas dentro del campo escogido de la medicina, para adquirir experiencia y dominio de la disciplina. La autoridad responsable del programa de formación debería tener los recursos para planificar e introducir los métodos de formación, evaluación de los residentes y las innovaciones del programa de formación. Debería haber representación del personal, de los residentes y de otros agentes relevantes en el diseño del programa formativo.

\section{Anotaciones:}

Otros agentes relevantes incluirían otros participantes en el proceso de formación, representantes de otros profesionales y autoridades sanitarias.

\section{EVALUACIÓN DE LOS RESIDENTES}

\subsection{MÉTODOS DE EVALUACIÓN}

\section{Estándares básicos}

La formación médica de postgrado debe incluir un proceso de evaluación, y las autoridades competentes deben definir y establecer los métodos utilizados para la evaluación de los residentes, incluyendo los criterios para superar los exámenes y otros tipos de evaluación. La evaluación debe hacer énfasis en los métodos formativos dentro de la residencia y en el feed-back constructivo.

\section{Desarrollo de la calidad}

La fiabilidad y validez de los métodos de evaluación deberían ser documentados y valorados, y se debería estimular a usar examinadores externos. Se debería aplicar un juego complementario de métodos evaluativos. Los diferentes estadios de la formación deberían documentarse en un libro del residente. Se debería establecer un mecanismo de revisión de exámenes, y cuando fuera necesario se debería proporcionar una segunda opinión, un cambio de tutor/supervisor o una formación complementaria.

\section{Anotaciones:}

- La definición de los métodos utilizados para evaluar incluye consideraciones sobre el balance entre la evaluación formativa y la sumativa, el número de exámenes y de otros tests, el balance entre diferentes tipos de exámenes, el uso de valoración normativa y de criterios (juicios referenciados), y el uso del portafolio y de tipos especiales de exámenes, p.e.exámenes clínicos objetivos y estructurados (ECOES).

- La valoración de los métodos de evaluación puede incluir consideraciones sobre como las evaluaciones promueven la formación y el aprendizaje.

- Los examinadores externos o auditores podrían aportar una perspectiva global.

\subsection{RELACIÓN ENTRE LA EVALUACIÓN Y LA FORMACIÓN}

\section{Estándares básicos}

Los principios, los métodos y las prácticas de evaluación deben ser claramente compatibles con los objetivos de formación y deben promover el aprendizaje. La evaluación debe documentar la adecuación de la formación.

\section{Desarrollo de la calidad}

Los métodos y las prácticas de evaluación deberían estimular un aprendizaje integrado y deberían evaluar requerimientos prácticos predefinidos, así como también conocimientos, aptitudes y actitudes. Los métodos usados deberían estimular una interacción constructiva entre la práctica clínica y la evaluación.

\subsection{FEEDBACK A LOS RESIDENTES}

\section{Estándares básicos}

Se debe dar un feed-back constructivo sobre la actuación del residente de forma regular.

\section{Desarrollo de la calidad}

Se debería especificar explícitamente los estándares aceptables de actuación, y aplicarlos a los residentes y a los supervisores. 


\section{Anotaciones:}

El feedback incluiría los resultados de la evaluación y los diálogos planificados sobre la actuación clínica, entre los residentes y los tutores/supervisores, con el objetivo de garantizar las instrucciones y los cambios necesarios para mejorar el desarrollo de las competencias.

\section{RESIDENTES}

\subsection{POLÍTICAS DE ADMISIÓN Y SELECCIÓN}

\section{Estándares básicos}

Las autoridades competentes y las organizaciones médicas profesionales deben ponerse de acuerdo sobre los criterios y el proceso de selección de residentes y los deben publicar y aplicar.

\section{Desarrollo de la calidad}

La política de selección debería definir criterios que considerasen las capacidades específicas de los potenciales residentes para mejorar el resultado del proceso de formación en el campo escogido de la medicina. El proceso de selección debería ser transparente y la admisión abierta a todos los licenciados en educación médica básica. El proceso de selección debería incluir un mecanismo de seguimiento y de apelación.

\section{Anotaciones:}

- El proceso de selección de los residentes incluiría racionalidad y métodos del proceso de selección y podría incluir la descripción de un mecanismo de revisión de examen.

- El seguimiento del proceso de admisión incluiría la mejora de los criterios de selección, para reflejar la capacidad de los residentes para ser competentes y para cubrir los cambios en las competencias necesarias relacionadas con la diversidad del campo escogido de la medicina.

- Los criterios de selección incluirían la consideración de una admisión equilibrada de acuerdo con el sexo, la raza y otros requerimientos sociales, incluyendo la necesidad potencial de una política de admisión para grupos de médicos menos privilegiados.

\subsection{NÚMERO DE RESIDENTES}

\section{Estándares básicos}

El número de residentes debe estar relacionado con las oportunidades de formación clínicas / prácticas, la capacidad de supervisión y otros recursos disponibles con el fin de garantizar una formación y una docencia de una calidad adecuada.

\section{Desarrollo de la calidad}

El número de residentes debería ser consultado con los agentes sociales relevantes. Siendo conscientes de la dificultad de predicción inherente a los recursos médicos en los diferentes ámbitos de la medicina, el número de puestos de formación debería ir cambiando periódicamente para ajustarse a las necesidades existentes de la comunidad, la sociedad y las fuerzas del mercado.

\section{Anotaciones:}

- Los agentes sociales serían aquellos responsables de planificar y desarrollar los recursos humanos en el sector sanitario local y nacional.

- La predicción de las necesidades de médicos formados por parte de la comunidad y la sociedad incluye la estimación de diversos mercados y factores demográficos así como también el desarrollo científico, los modelos migratorios de los médicos, etc.

\subsection{SOPORTE Y CONSEJO A LOS RESIDENTES}

\section{Estándares básicos}

Las autoridades competentes, en colaboración con la profesión, deben garantizar un sistema que facilite el acceso al soporte, al consejo y a la orientación profesional de los residentes.

\section{Desarrollo de la calidad}

El dar consejo debería estar basado en el seguimiento del progreso en la formación y de la información sobre problemas que puedan surgir, y debería estar dirigido a satisfacer las necesidades sociales y personales de los residentes.

\section{Anotaciones:}

Las necesidades sociales y personales incluirían el soporte profesional, los problemas de salud, los de vivienda y los problemas económicos.

\subsection{CONDICIONES DE TRABAJO}

\section{Estándares básicos}

La formación de postgrado debe llevarse a cabo en puestos remunerados apropiadamente en la especialidad escogida de la medicina y debe incluir la participación en todas las actividades médicas relevantes para la formación, incluyendo guardias, dedicando así las actividades profesionales a la formación práctica y el tiempo estándar de trabajo al aprendizaje teórico. Las condiciones de servicio y las responsabilidades de los residentes deben estar definidas y deben darse a conocer a todos los implicados. 


\section{Desarrollo de la calidad}

Los cargas del servicio de residente no deberían ser excesivas y la estructura de las guardias debería tener en cuenta las necesidades de los pacientes, la continuidad en la atención y las necesidades educativas del residente. Se debería permitir la formación a tiempos parcial bajo circunstancias especiales, determinadas por las autoridades competentes y estructuradas de acuerdo con un programa hecho a medida. La duración total y la calidad de la formación a tiempo parcial no deberían de ser inferiores a aquellas a tiempo total. La interrupción de la formación por razones como el embarazo (incluyendo la baja por maternidad o paternidad), enfermedad o servicio militar deberían ser reemplazadas por formación adicional.

\section{Anotaciones:}

- Las posiciones de servicio contractual incluirían médico interno, residente, becario, etc.

- Los componentes del servicio de los residentes han de estar sujetos a las definiciones y protecciones intrínsecas del contrato.

\subsection{REPRESENTACIÓN DE LOS RESIDENTES}

\section{Estándares básicos}

Debe existir una política sobre representación de los residentes y la participación apropiada en el diseño y evaluación del currículum, las condiciones de trabajo así como en otros asuntos relevantes para los residentes.

\section{Desarrollo de la calidad}

Se debería animar las organizaciones de residentes a tomar decisiones sobre los procesos de formación, y las condiciones y regulaciones.

\section{Anotaciones:}

La representación de los residentes incluiría la participación en grupos o comités responsables del diseño del programa a nivel local o nacional.

\section{PERSONAL ACADÉMICO}

\subsection{POLÍTICA DE CONTRATACION}

\section{Estándares básicos}

La política sobre contratación de los formadores, supervisores y profesores debe especificar la experteza necesaria y sus tareas y responsabilidades. Esta política debe especificar las obligaciones del personal formador y específicamente el balance entre funciones educativas, de servicio y otros deberes.

\section{Desarrollo de la calidad}

Todos los médicos deberían, como parte de sus obligaciones profesionales, reconocer su responsabilidad en participar en la formación postgraduada de los médicos basada en la práctica. La participación en la formación postgraduada debería ser reconocida. La política de personal debería garantizar que los formadores estuvieran siempre al día en su especialidad, en toda su extensión, y los formadores subespecializados solo fuesen aprobados para períodos específicos relevantes durante la formación.

\section{Anotaciones:}

- La experteza incluiría el reconocimiento como un especialista en el campo relevante de la medicina.

- El personal formador incluiría médicos y otros profesionales sanitarios.

- Las otras tareas incluirían funciones administrativas así como otras responsabilidades educativas o de investigación.

\subsection{OBLIGACIONES Y DESARROLLO DE LOS FORMADORS}

\section{Estándares básicos}

En las agendas de trabajo de los formadores deben incluirse actividades de instrucción como responsabilidad y se debe describir su relación con las agendas de los residentes.

\section{Desarrollo de la calidad}

La política de personal debería incluir el soporte a los formadores incluyendo formación y más desarrollo si hiciera falta, y debería valorar y reconocer las actividades académicas meritorias, incluyendo las funciones como formadores, supervisores y profesores. La proporción entre el número de formadores reconocidos y el número de residentes debería garantizar una interacción personal cercana y un seguimiento del residente.

\section{Anotaciones:}

El reconocimiento de actividades académicas meritorias se haría mediante premios, ascensos y/o remuneraciones.

\section{CENTROS DE FORMACIÓN Y RECURSOS EDUCATIVOS}

\subsection{INSTALACIONES Y PACIENTES}

\section{Estándares básicos}

Los centros para realizar la formació deben ser seleccionados y reconocidos por las autoridades competentes y deben tener suficientes instalaciones clínico-prácticas para facilitar la formación. Estos centros para la forma- 
ción deben de tener un número suficiente de pacientes y una variedad de casos apropiada para cubrir los objetivos de la formación. La formación debe exponer al residente a un abanico amplio de experiencia en la especialidad escogida y, cuando sea relevante, debe incluir tanto pacientes ingresados como pacientes atendidos en consultas externas ambulatorias y guardias.

\section{Desarrollo de la calidad}

El número de pacientes y la variedad de casos debería ofrecer una experiencia clínica en todos los aspectos de le especialidad escogida, incluyendo la formación en la promoción de la salud y la prevención de las enfermedades. La formación debería llevarse a cabo en hospitales universitarios, y cuando conviniera, parte de la formación debería llevarse a cabo en otros hospitales/instituciones relevantes y centros de la comunidad. Debería realizarse regularmente una evaluación de las calidad de las instalaciones implicadas en la formación.

\section{Anotaciones:}

- Las instalaciones basadas en la comunidad incluirían las consultas de especialistas, las clínicas de especialistas, las residencias geriátricas, los centros de atención primaria y otros lugares donde se presta atención sanitaria.

- La calidad de las instalaciones para la formación puede ser evaluada mediante visitas a los referidos centros.

\subsection{INSTALACIONES FÍSICAS Y EQUIPAMIENTO}

\section{Estándares básicos}

El residente debe tener espacio y oportunidades para la práctica y el estudio teórico y debe tener acceso a la literatura profesional adecuada así como también a equipamiento para la formación en técnicas prácticas.

\section{Desarrollo de la calidad}

Se deberían evaluar periódicamente las instalaciones físicas y el equipamiento para garantizar su adecuación y calidad para la formación de postgrado.

\section{Anotaciones:}

Las instalaciones físicas de los centros de formación incluirían aulas, salas de seminarios, laboratorios, bibliotecas, equipamiento informático y zonas de descanso.

\subsection{EQUIPOS CLÍNICOS}

\section{Estándares básicos}

La formación clínica debe incluir experiencia de trabajo en grupo con colegas y otros profesionales sanitarios.

\section{Desarrollo de la calidad}

El proceso de formación debería permitir el aprendizaje en equipos multidisciplinarios, resultando en la capacidad para trabajar eficazmente con colegas y otros profesionales sanitarios como miembro o líder del equipo sanitario, y debería desarrollar para dirigir y enseñar a otros profesionales sanitarios.

\subsection{TECNOLOGÍA DE LA INFORMACIÓN}

\section{Estándares básicos}

Debe haber una política que incluya el uso eficaz de la tecnología de la información y la comunicación en el programa de formación, con el objetivo de garantizar una atención adecuada del paciente.

\section{Desarrollo de la calidad}

Los formadores y los residentes deberían ser competentes en el uso de la informática para el autoaprendizaje, el acceso de información, y para trabajar en los sistemas sanitarios.

\section{Anotaciones:}

- Una política sobre el uso de ordenadores, redes internas y externas y otros sistemas de información y comunicación, debería incluir la coordinación con los servicios de biblioteca de la institución.

- El uso de la tecnología de la información y de la comunicación puede ser parte de la formación en la medicina basada en la evidencia y en la preparación de los residentes para la educación médica continuada y el desarrollo profesional.

\subsection{INVESTIGACIÓN}

\section{Estándares básicos}

Debe haber una política que fomente la integración de la práctica y la investigación en los centros de formación. Los centros de formación deben disponer de instalaciones para a la investigación y para actividades y prioridades de investigación.

\section{Desarrollo de la calidad}

Debería haber oportunidades para combinar la formación clínica con la investigación. Se debería estimular a los residentes para que participasen en procesos de desarrollo de la calidad en salud e investigación.

\subsection{EXPERTEZA EDUCATIVA}

\section{Estándares básicos}

Debe haber una política sobre el uso de la experteza 
educativa relevante para planificar, desarrollar y evaluar la formación.

\section{Desarrollo de la calidad}

Se debería disponer de acceso a expertos educativos, y se debería ser consciente de la importancia que tiene utilizar a estos expertos para el desarrollo del personal docente y para la investigación en la educación médica de postgrado.

\section{Anotaciones:}

- La experteza educativa se encargaría de los problemas, los procesos y la práctica de la formación y la evaluación médica de postgrado, e incluiría médicos con experiencia en educación médica, psicólogos educadores, sociólogos, etc. La experteza educativa puede ubicarse en una unidad educativa de la institución o ser obtenida de otra institución nacional o internacional.

- La investigación en educación médica estudia la efectividad de los métodos de enseñanza y aprendizaje, así como el contexto institucional en el sentido más amplio.

\subsection{INTERCAMBIOS EDUCATIVOS}

\section{Estándares básicos}

Debe haber una política de acceso a las oportunidades de formación individual en otros centros del mismo país o del extranjero, que cumplan con los requerimientos para completar la formación y para posibilitar la transferencia de los créditos de formación.

\section{Desarrollo de la calidad}

El intercambio regional o internacional de personal académico y residentes debería ser facilitado dotándolo de los recursos adecuados. Las autoridades competentes deberían establecer relaciones con los organismos correspondientes nacionales e internacionales con el objetivo de facilitar el intercambio y el reconocimiento mutuo de elementos formativos.

\section{Anotaciones:}

La transferencia de créditos formativos se puede facilitar mediante la coordinación activa de programas entre instituciones formativas.

\section{EVALUACIÓN DE LA FORMACIÓN}

\subsection{MECANISMOS PARA LA EVALUACIÓN DE LOS PROGRAMAS EDUCATIVOS}

\section{Estándares básicos}

Las autoridades relevantes y la profesión deben esta- blecer un mecanismo para la evaluación del programa de formación que permita realizar un seguimiento del proceso de formación, de las instalaciones y del progreso del residente, y que garantice que los problemas son identificados y solucionados.

\section{Desarrollo de la calidad}

La evaluación del programa debería incluir el contexto de los procesos de formación, la estructura y componentes específicos del programa y los resultados generales que se esperan.

\section{Anotaciones:}

- Los mecanismos para la evaluación del programa implicaría el uso de métodos válidos y fiables y convendría que la información básica sobre el programa de formación estuviera disponible. La implicación de expertos en educación médica faciltaría la base de la evidencia de la calidad de la formación de postgrado.

- Como problemas identificados se incluirían problemas presentados a los comités de programa, formadores y tutores, etc.

- El contexto de los procesos educativos incluiría la organización y recursos así como también el entorno de aprendizaje.

- Los componentes específicos para la evaluación del currículum incluirían la descripción del programa de formación y la actuación de los residentes.

- Los resultados generales se podrían medir por ejemplo a partir de la elección de especialidad y el progreso de los residentes.

\subsection{FEEDBACK DE LOS FORMADORES Y LOS RESIDENTES}

\section{Estándares básicos}

Se debe obtener sistemáticamente información sobre la calidad del programa, a partir de las opiniones de los profesores y residentes y actuar en consecuencia.

\section{Desarrollo de la calidad}

Se debería implicar activamente a los formadores y residentes en la planificación de la evaluación del programa y en el uso de sus resultados para el desarrollo del mismo.

\section{Anotaciones:}

El feedback sobre el programa incluiría informes de los residentes sobre las condiciones en sus cursos. 


\subsection{PROGRESO DE LOS RESIDENTES}

\section{Estándares básicos}

El progreso de los residentes debe ser evaluado en relación con el proceso de formación y de la misión de la educación médica de postgrado.

\section{Desarrollo de la calidad}

El progreso de los residentes debería ser analizada en relación con su experiencia y a sus calificaciones de entrada, y se debería utilizar para dar feedback a los comités responsables de la selección de residentes, de la planificación del programa y el asesoramiento.

\section{Anotaciones:}

Las medidas del progreso de los residentes incluirían la información sobre la duración media de la formación, los resultados, la proporción entre aptos y no aptos en las pruebas, proporción de éxito y de abandono, así como también el tiempo que los residentes han dedicado a áreas de especial interés.

\subsection{ACREDITACIÓN Y SEGUIMIENTO DE LOS CENTROS DE FORMACIÓN}

\section{Estándares básicos}

Todos los programas de formación deben estar acreditados por una autoridad competente que actúe según un criterio bien definido y una evaluación del programa y con la capacidad de conceder o, si es el caso, retirar el reconocimiento de los centros de formación o de los cursos teóricos.

\section{Desarrollo de la calidad}

Las autoridades competentes deberían establecer un sistema de seguimiento de los centros de formación y de otras instalaciones educativas mediante visitas a los centros ú otros procedimientos relevantes.

\section{Anotaciones:}

Los criterios para acreditar centros de formación incluiría valores mínimos de número y diversidad de pacientes, equipamiento, biblioteca e instalaciones informáticas, personal de formación y programa de formación.

\subsection{IMPLICACIÓN DE LOS DIFERENTES AGENTES SOCIALES}

\section{Estándares básicos}

El proceso y los resultados de la evaluación deben implicar a los gestores y a la administración de los centros de formación, a los formadores y a los residentes y deben ser transparentes para todos los agentes sociales.

\section{Desarrollo de la calidad}

Los procesos y los resultados de las evaluaciones deben ser creíbles por los principales agentes sociales implicados.

\section{Anotaciones:}

- Los agentes sociales incluirían las organizaciones profesionales médicas, otras profesiones sanitarias, autoridades sanitarias y autoridades implicadas en la formación de los médicos y personal paramédico, gerentes de hospitales y proveedores de atención primaria, pacientes y organizaciones de pacientes.

- Los agentes sociales principales incluirían formadores, residentes y autoridades sanitarias.

\section{GOBERNABILIDAD Y GESTIÓN}

\subsection{GOBERNABILIDAD}

\section{Estándares básicos}

La formación debe dirigirse de acuerdo con las normativas sobre estructura, contenido, proceso y resultados, elaboradas por las autoridades competentes. Se debe documentar la realización de la formación mediante calificaciones, diplomas, certificados u otra evidencia de calificación formal, conferidas como la base para el reconocimiento formal del médico competente en el campo médico escogido, por las autoridades correspondientes. La autoridad competente debe evaluar continuadamente los programas de formación, las instituciones y los formadores. La autoridad competente debe ser responsable de iniciar un programa para la formación cualitativa.

\section{Desarrollo de la calidad}

Se deberían desarrollar procedimientos documentados que puedan verificar la realización de la formación para uso de las autoridades nacionales e internacionales.

\section{Anotaciones:}

El reconocimiento de un médico como competente incluiría, dependiendo del nivel de la formación, médicos con derecho a ejercer una práctica independiente, especialistas, subespecialistas, expertos, etc.

\subsection{LIDERAZGO PROFESIONAL}

\section{Estándares básicos}

Las responsabilidades del líder profesional para la educación médica de postgrado deben estar claramente definidas.

\section{Desarrollo de la calidad}

El líder profesional debería ser evaluado de forma 
periódica, en relación al cumplimiento de los objetivos y los resultados de la educación médica de postgrado.

\subsection{PRESUPUESTO EDUCATIVO Y DISTRIBUCIÓN DE RECURSOS}

\section{Estándares básicos}

Debe haber una línea clara de responsabilidad y autoridad para presupuestar los recursos destinados a formación.

\section{Desarrollo de la calidad}

El presupuesto se debería distribuir de forma que diese soporte a la misión y a los objetivos de los programas de formación y del servicio.

\section{Anotaciones:}

El presupuesto de los recursos de formación dependería de la práctica presupostaria en cada institución y país.

\subsection{ADMINISTRACIÓN}

\section{Estándares básicos}

El personal administrativo de los programas de formación médicos de postgrado debe ser apropiado para la aplicación del programa, y para garantizar una buena administración y desplegamiento de sus recursos.

\section{Desarrollo de la calidad}

La administración debería incluir un programa de garantía de calidad, cuya gestión debería ser revisada regularmente para alcanzar la mejora de la calidad.

\subsection{REQUERIMIENTOS Y NORMATIVAS}

\section{Estándares básicos}

Debe haber un organismo nacional responsable de definir el número y el tipo de especialidades médicas reconocidas y otras funciones médicas expertas, para las cuales se desarrollen programas de formación aprobados.

\section{Desarrollo de la calidad}

La definición de programas de postgrado aprobados se debería hacer en colaboración con todos los agentes sociales relevantes implicados.

\section{Anotaciones:}

- Un organismo nacional establecido de acuerdo a las leyes nacionales y normativas, actuaría en interés de la sociedad globalmente.

- Los agentes relevantes incluirían autoridades sanitarias nacionales y locales, universidades, organizaciones profesionales médicas, el público, etc.

\section{RENOVACIÓN CONTINUADA}

\section{Estándares básicos}

En respuesta a la naturaleza dinámica de la formación médica de postgrado, las autoridades relevantes deben iniciar procedimientos para la revisión regular y la actualización de la estructura, función y calidad de los programas de formación, y deben corregir las deficiencias detectadas.

\section{Desarrollo de la calidad}

El proceso de renovación debería estar basado en estudios y análisis prospectivos y debería llevar a la revisión de las políticas y las prácticas de los programas de formación médica de postgrado de acuerdo con las experiencias acumuladas, las actividades actuales y las perspectivas futuras. Haciéndolo de esta manera, debería incluir los siguientes puntos:

- La adaptación de la misión y los objetivos de la formación de postgrado al desarrollo científico, socioeconómico y cultural de la sociedad.

- La modificación de las competencias necesarias al completar la formación de postgrado en el campo escogido de la medicina, de acuerdo con las necesidades del entorno en el que los nuevos médicos formados ejercerán.

- La adaptación de los métodos de aprendizaje y formación para garantizar que estos son apropiados y relevantes.

- La adecuación de la estructura, contenido y duración de los programas de formación para adaptarse a los avances en las ciencias biomédicas, las ciencias clínicas, las ciencias sociales y del comportamiento, los cambios en el perfil demográfico y del patrón salud / enfermedad de la población y de las condiciones culturales.

- El desarrollo de los principios y de los métodos evaluativos de acuerdo con los cambios en los objetivos de formación.

- Adaptación de la política de captación y de unos métodos de selección de residentes a las expectativas y circunstancias cambiantes, a las necesidades de recursos humanos, a los cambios en educación médica básica y a los requerimientos del programa de formación.

- Adaptación de la política de captación y de formación del personal docente (formadores, supervisores, profesores, etc) de acuerdo con las necesidades cambiantes en la formación de postgrado.

- Puesta al día de los centros de formación y de otros recursos educativos de acuerdo con las necesidades cambiantes en la formación de postgrado, p.e. el número de residentes, el número y el perfil de los formadores, el programa de formación y los principios contemporáneos de formación.

- Mejora del proceso de seguimiento y evaluación del programa.

- Desarrollo de la estructura organizativa y de gestión para adaptarse a las circunstancias cambiantes y las necesidades en la formación de postgrado y, con el paso del tiempo, acomodándose a los intereses de los diferentes agentes sociales. 